\title{
KONUS-WIND OBSERVATION OF THE ULTRA-LUMINOUS GRB 110918A
}

\author{
D. Frederiks ${ }^{1}$, D. Svinkin ${ }^{1,2}$, R. Aptekar ${ }^{1,2}$, S. Golenetskii ${ }^{1}$, E. Mazets ${ }^{1}$, \\ P. Oleynik ${ }^{1,2}$, V. Pal'shin ${ }^{1,2}$, A. Tsvetkova ${ }^{1}$, M. Ulanov ${ }^{1,2}$ and T. Cline ${ }^{3}$
}

\begin{abstract}
The exceptionally intense long GRB 110918A was discovered by several GRB observing missions: INTEGRAL (SPI-ACS), KonusWIND, Mars Odyssey (HEND), and MESSENGER (GRNS) on September 18, 2011. This GRB was localized by the Interplanetary Network (IPN) and its bright X-ray counterpart was found in close vicinity of the IPN box in the Swift/XRT follow-up observations starting 1.2 days after the trigger. The optical afterglow was discovered by the Isaac Newton Telescope and its spectroscopic redshift $\mathrm{z}=0.982$ was measured with the GMOS spectrograph mounted on the Gemini$\mathrm{N}$ telescope. GRB 110918A is the brightest burst detected by KonusWIND for more than 17 years of its continuous observations. The instrument's light curves in three energy bands covering $22-1450 \mathrm{keV}$ range show an extremely bright, short, hard pulse followed by three weaker, softer, partly overlapping pulses within next 25 seconds. A spectral lag between the light-curves is determined, showing a substantial increase in the course of the burst. The emission is detected up to $12 \mathrm{MeV}$. Modeling the time-integrated energy spectrum with the Band function yields a moderate value of $E_{\text {peak }}=340 \mathrm{keV}$, while the time-resolved spectral analysis reveals strong hardness-intensity correlation and a hard-to-soft evolution of the emission: $E_{\text {peak }}$ falls from $\sim 4 \mathrm{MeV}$ at the onset of the huge initial pulse to $\sim 50 \mathrm{keV}$ at the final stage of the burst. The total $20 \mathrm{keV}-10 \mathrm{MeV}$ energy fluence amounts to $S=(7.8 \pm 0.4) \times 10^{-4} \mathrm{erg} \mathrm{cm}^{-2}$ and a 64 -ms peak flux $F_{\text {max }}=(9.2 \pm 0.4) \times 10^{-4} \mathrm{erg} \mathrm{cm}^{-2} \mathrm{~s}^{-1}$, which corresponds to a huge isotropic-equivalent energy release $E_{\text {iso }}=(2.1 \pm 0.1) \times 10^{54} \mathrm{erg}$ and the record-breaking peak luminosity $L_{\text {iso; } \max }=(4.7 \pm 0.2) \times 10^{54} \mathrm{erg} \mathrm{s}^{-1}$.
\end{abstract}

1 Ioffe Physico-Technical Institute, St. Petersburg 194021, Russia

2 St. Petersburg State Technical University, St. Petersburg 195251, Russia

3 Emeritus: NASA's Goddard Space Flight Center, Greenbelt, MD 20771, USA 

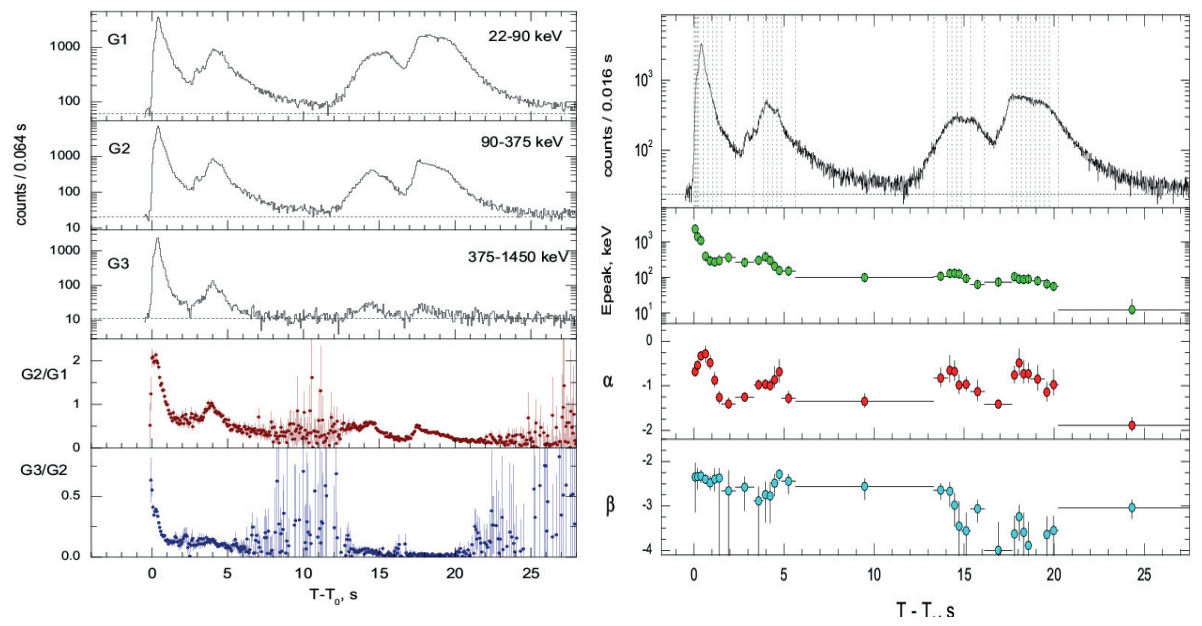

Fig. 1. Left: the light curves of GRB 110918A recorded by Konus- WIND in three energy bands (three upper panels) and their hardness ratios evolution (two lower panels). Right: the temporal behavior of the Band spectral model parameters $\left(E_{\text {peak }}, \alpha, \beta\right)$ obtained from the time-resolved fits. The light curve in the $22-1450 \mathrm{keV}$ band is given for the reference in the upper panel.

\section{Introduction}

The exceptionally intense long GRB 110918A was discovered by several GRB observing missions: INTEGRAL (SPI-ACS), Konus-WIND, Mars Odyssey (HEND), and MESSENGER (GRNS) on Sep. 18, 2011. This burst source was localized by the Interplanetary Network (Hurley et al. 2011) and its bright X-ray counterpart was found in close vicinity of the IPN box in the Swift/XRT follow-up observations starting 1.2 days after the trigger (Mangano et al. 2011). The optical afterglow was discovered by the Isaac Newton Telescope (Tanvir et al. 2011) and its spectroscopic redshift $\mathrm{z}=0.982$ was measured with the GMOS spectrograph mounted on the Gemini-N telescope (Levan et al. 2011). A preliminary analysis of the Konus-WIND observation revealed that GRB 110918A is the most intense gamma-ray burst among 2200 GRBs observed by the instrument since November, 1994 (Golenetskii et al. 2011). The high energy flux also suggests enormous isotropic-equivalent rest-frame energy (Frederiks \& Pal'shin 2011). These preliminary estimates place GRB 110918A among the brightest GRBs ever observed in the era of cosmological GRBs and this burst deserves, without a doubt, a detailed consideration.

\section{Time histories}

GRB 110918A triggered the Konus-WIND $\gamma$-ray spectrometer (Aptekar et al. $1995)$ at $T_{0}=77222.856 \mathrm{~s}$ UT $(21: 27: 02.856)$ on September 18, 2011. In the triggered mode, count rates are recorded in three energy bands: G1(22-90 keV), 
G2(90-375 keV), and G3(375-1450 keV) with an accumulation time varying from 2 to $256 \mathrm{~ms}$. The burst light curve in the three energy bands (Fig. 1, left) is clearly divided into two groups of pulses $\left(T_{0}-T_{0}+12 \mathrm{~s}\right)$ and $\left(T_{0}+12 \mathrm{~s}-T_{0}+28 \mathrm{~s}\right)$, each, in turn, having two pronounced peaks As shown in the same Figure, the temporal evolution of the G2/G1 and G3/G2 hardness ratios indicates an apparent hardness-intensity correlation of the emission against a general tendency of spectral softening in the course of the event. In the G2+G3 energy band (90-1450 keV), the duration $T_{90}$ is $19.6 \pm 0.1 \mathrm{~s}$ and $T_{50}=14.3 \pm 0.1 \mathrm{~s}$. The peak $64-\mathrm{ms}$ count rate reached is $(1.46 \pm 0.02) \times 10^{5}$ counts $/ \mathrm{s}$ in the bin starting at $\mathrm{T}_{0}+0.368 \mathrm{~s} \mathrm{We}$ examined the spectral lag using the cross-correlation function between the 16-ms G1, G2, and G3 light curves at different phases of the burst The statistically significant positive lags of 40-400 ms are derived for both the first $\left(T_{0}-T_{0}+12 \mathrm{~s}\right)$ and the second $\left(T_{0}+12-T_{0}+25 \mathrm{~s}\right)$ groups of pulses However, for the second group of pulses the observed lags are 2-4 times longer than for the initial phase of the event.

\section{Spectral analysis and energetics in gamma-rays}

During the main phase of GRB 110918A, 35 energy spectra were recorded in 128 quasi-log channels of two overlapping energy bands PHA1 (20-1450 keV) and PHA2 (375 keV-14 MeV). Their accumulation time is varied from $0.064 \mathrm{~s}$ to $8.192 \mathrm{~s}$, depending on the current intensity of the burst, the emission is seen up to $12 \mathrm{MeV}$. The spectral analysis was performed with XSPEC, version 12.5. A good quality of the fit with the Band GRB function is achieved for the majority of the spectra, which enabled us to construct the temporal behavior of the model parameters $\alpha, \beta, E_{\text {peak }}$ (Fig. 1, right). The time-resolved spectral analysis confirms strong hardness-intensity correlation and a hard-to-soft evolution of the emission: the low-energy photon index $\alpha$ evolution is strongly correlated with the burst intensity, and $E_{\text {peak }}$ falls from $\sim 4 \mathrm{MeV}$ at the onset of the huge initial pulse to $\sim 50 \mathrm{keV}$ at the final stage of the burst.

The spectrum at the culmination of the initial pulse is described by $\alpha=-0.33 \pm$ 0.09 (90\% conf.), $\beta=-2.3 \pm 0.1$, and $E_{\text {peak }}=1080 \pm 150 \mathrm{keV}\left(\chi^{2}=0.67\right.$, 68 dof). We analyzed the time-averaged spectrum of the whole burst and its separate phases. It should be emphasized that, having $E_{\text {peak }}=340 \pm 60 \mathrm{keV}$, $\alpha=-1.65 \pm 0.05$, and $\beta=-2.25 \pm 0.09$ ( $\chi^{2}=0.96,81$ dof $)$ the overall timeintegrated spectrum is, indeed, an "average" one and doesn't reflect the strong spectral evolution. As it is expected from the time-resolved spectral analysis, the average spectra of the first $\left(T_{0}-T_{0}+13.312 \mathrm{~s}\right)$ and the second $\left(T_{0}+13.312-T_{0}+\right.$ $28.416 \mathrm{~s}$ ) groups of pulses are strongly different. With only the low-energy photon index being close $(\alpha \sim-1.2)$, the values of $E_{\text {peak }}$ differ almost by an order of magnitude $(630 \mathrm{keV}$ and $80 \mathrm{keV}$, respectively), and the high-energy photon index $\beta$ is sufficiently harder for the first group of pulses $(-2.3$ vs. -3.3 for the second group).

Based on the results of our spectral and temporal analyses, we calculated the observed energetics of GRB 110918A in gamma-rays. The $20 \mathrm{keV}-10 \mathrm{MeV}$ energy 
fluence $\mathrm{S}$ measured from $T_{0}$ to $T_{0}+28.416 \mathrm{~s}$ amounts to $(7.8 \pm 0.5) \times 10^{-4} \mathrm{erg} \mathrm{cm}^{-2}$. In the same energy range, the 64 -ms peak energy flux $F_{\max }=(9.2 \pm 0.4) \times$ $10^{-4} \mathrm{erg} \mathrm{cm}^{-2} \mathrm{~s}^{-1}$ in the interval beginning at $T_{0}+0.384 \mathrm{~s}$, at the culmination of the huge initial pulse.

Assuming the redshift $\mathrm{z}=0.982$ (Levan et al. 2011) and a standard cosmology with $\mathrm{H}_{0}=71 \mathrm{~km} / \mathrm{s} / \mathrm{Mpc}, \Omega_{\mathrm{M}}=0.27$, and $\Omega_{\lambda}=0.73$, we derive the following restframe parameters of the prompt gamma-ray emission: the isotropic-equivalent energy release $E_{\text {iso }}=(2.1 \pm 0.1) \times 10^{54} \mathrm{erg}$, the peak luminosity $L_{\text {iso } \max }=$ $(4.7 \pm 0.2) \times 10^{54} \mathrm{erg} / \mathrm{s}$ (both in the bolometric $1-10000 \mathrm{keV}$ energy range), and the intrinsic peak energy $E_{p, i}=680 \pm 140 \mathrm{keV}$. These estimations make GRB 110918A the most luminous gamma-ray burst ever observed in the cosmological era.

\section{Summary}

The detailed analysis of the ultra-luminous GRB 110918A observation by the Konus-WIND gamma-ray spectrometer reveals that the burst is the brightest among $\sim 2200$ GRBs recorded by the instrument since November, 1994. The record-breaking values of the peak count rate, peak energy flux, and the total energy fluence are obtained. Also, assuming the redshift $\mathrm{z}=0.982$, this burst is the most luminous GRB ever observed since the start of the era of cosmological GRB studies in 1997. The temporal behavior of the light curves hardness ratios, the obtained spectral lags, and the results of time-resolved spectral fits show an apparent hardness-intensity correlation of the observed emission and a general spectral softening in the course of the burst.

The detailed coverage of the Konus- WIND results on GRB 110918A as well as the refined IPN localization, the X-ray afterglow observations with Swift/XRT, and the optical monitoring with Swift/UVOT can be found in the forthcoming paper (Frederiks, Hurley et al., in preparation).

This work was supported by a Russian Space Agency contract the Russian Foundation for Basic Research projects 11-02-12082-ofi-m-2011 and 12-02-00032-a, and the Ministry of Education and Science of Russian Federation contract \#11.G34.31.0001 with SPbSPU and leading scientist G.G. Pavlov.

\section{References}

Aptekar, R.L., Frederiks, D.D., Golenetskii, S.V., et al., 1995, Space Sci. Rev., 71, 265 Frederiks, D., \& Pal'shin, V., 2011, GRB Coordinates Network, 12370, 1

Golenetskii, S., Aptekar, R., Frederiks, D., et al., 2011, GRB Coordinates Network, 12362,1

Hurley, K., Golenetskii, S., Aptekar, R., et al., 2011, GRB Coordinates Network, 12357, 1 Levan, A.J., Tanvir, N.R., Wiersema, K., et al., 2011, GRB Coordinates Network, 12368,1

Mangano, V., Sbarufatti, B., Evans, P., \& Krimm, H., 2011, GRB Coordinates Network, 12364,1

Tanvir, N.R., Wiersema, K., Levan, A.J., et al., 2011, GRB Coordinates Network, 12365,1 